\title{
Nasal Expiratory Positive Airway Pressure Devices (Provent) for OSA: A Systematic Review and Meta-Analysis
}

\author{
Muhammad Riaz, ${ }^{1}$ Victor Certal, ${ }^{2,3}$ Gaurav Nigam, ${ }^{4}$ Jose Abdullatif, ${ }^{5}$ Soroush Zaghi, \\ Clete A. Kushida, ${ }^{7}$ and Macario Camacho ${ }^{7,8}$ \\ ${ }^{1}$ Twin Cities Community Hospital, Templeton, CA 93465, USA \\ ${ }^{2}$ Department of Otorhinolaryngology, Sleep Medicine Centre, Hospital CUF, 4100-180 Porto, Portugal \\ ${ }^{3}$ Centre for Research in Health Technologies and Information Systems (CINTESIS), University of Porto, 4200-450 Porto, Portugal \\ ${ }^{4}$ Clay County Hospital, 911 Stacy Burk Drive, Flora, IL 62839, USA \\ ${ }^{5}$ Department of Otorhinolaryngology, Hospital Bernardino Rivadavia, Buenos Aires, Argentina \\ ${ }^{6}$ Department of Head and Neck Surgery, University of California, Los Angeles, CA 90095, USA \\ ${ }^{7}$ Sleep Medicine Division, Department of Psychiatry and Behavioral Sciences, Stanford Hospital and Clinics, Redwood City, \\ CA 94063, USA \\ ${ }^{8}$ Division of Otolaryngology, Sleep Surgery, and Sleep Medicine, Tripler Army Medical Center (Tripler AMC), 1 Jarrett White Road, \\ Honolulu, HI 96859, USA \\ Correspondence should be addressed to Muhammad Riaz; hmriazmalik08@gmail.com
}

Received 20 September 2015; Revised 18 November 2015; Accepted 19 November 2015

Academic Editor: Giora Pillar

Copyright (C) 2015 Muhammad Riaz et al. This is an open access article distributed under the Creative Commons Attribution License, which permits unrestricted use, distribution, and reproduction in any medium, provided the original work is properly cited.

\begin{abstract}
Objective. To quantify the effectiveness of nasal expiratory positive airway pressure (nasal EPAP) devices or Provent as treatment for obstructive sleep apnea (OSA). Methods. PubMed and six other databases were searched through November 15, 2015, without language limitations. Results. Eighteen studies (920 patients) were included. Pre- and post-nasal EPAP means \pm standard deviations $(\mathrm{M} \pm \mathrm{SD})$ for apnea-hypopnea index $(\mathrm{AHI})$ in 345 patients decreased from $27.32 \pm 22.24$ to $12.78 \pm 16.89$ events/hr (relative reduction $=53.2 \%)$. Random effects modeling mean difference $(\mathrm{MD})$ was -14.78 events/hr $[95 \% \mathrm{CI}-19.12,-10.45], p$ value $<$ 0.00001. Oxygen desaturation index (ODI) in 247 patients decreased from $21.2 \pm 19.3$ to $12.4 \pm 14.1$ events/hr (relative reduction $=$ $41.5 \%, p$ value $<0.00001$ ). Lowest oxygen saturation (LSAT) $\mathrm{M} \pm$ SD improved in 146 patients from $83.2 \pm 6.8 \%$ to $86.2 \pm 11.1 \%, \mathrm{MD}$ 3 oxygen saturation points [95\% CI 0.57, 5.63]. Epworth Sleepiness Scale (ESS) M \pm SD improved (359 patients) from $9.9 \pm 5.3$ to 7.4 $\pm 5.0, \mathrm{MD}-2.5$ [95\% CI $-3.2,-1.8]$, $p$ value $<0.0001$. Conclusion. Nasal EPAP (Provent) reduced AHI by $53.2 \%$, ODI by $41.5 \%$ and improved LSAT by 3 oxygen saturation points. Generally, there were no clear characteristics (demographic factors, medical history, and/or physical exam finding) that predicted favorable response to these devices. However, limited evidence suggests that high nasal resistance could be associated with treatment failure. Additional studies are needed to identify demographic and polysomnographic characteristics that would predict therapeutic success with nasal EPAP (Provent).
\end{abstract}

\section{Introduction}

Obstructive sleep apnea (OSA) is a chronic disorder affecting millions of Americans with an escalating prevalence between $3 \%$ and $10 \%$ in middle-aged adults [1]. Positive airway pressure (PAP) is considered as the most effective treatment for OSA [2]. Consistent PAP device usage leads to improvement or even complete alleviation of daytime symptoms and PAP therapy also mitigates cardio- and neurovascular complications of untreated sleep apnea [3]. Nevertheless, poor PAP adherence is considered as a major barrier for optimal OSA treatment [4]. Therefore alternative treatment options have been investigated over the years to include upper airway surgeries, hypoglossal nerve stimulators, oral appliances, and 
Winx oral devices as well as more recently Provent therapy which are also known as nasal expiratory positive airway pressure (nasal EPAP) devices.

Nasal EPAP device consists of disposable one-way resister valves, which are placed over the nostrils with an adhesive tape. These valves operate by utilizing the patient's own breathing to create a positive end-expiratory pressure with minimal inspiratory resistance. This high-end-expiratory pressure leads to upper airway dilation with subsequent tracheal traction and increased lung volumes during exhalation, thereby making the upper airway more resistant to ensuing inspiration [5-7]. Several studies have evaluated the efficacy of nasal EPAP given high noncompliance rate of PAP therapy due to either intolerance or difficulty associated with its use during travelling, in wilderness, and in places with lack of electricity [8]. The objective of this systematic review and meta-analysis is to quantitatively evaluate the effectiveness of nasal EPAP on polysomnography variables and sleepiness in OSA patients.

\section{Methods}

This study is exempt from Institutional Review Board (IRB) protocol being a systematic review and meta-analysis. Electronic databases were searched initially from inception through February 1, 2015, with an update through November 15, 2015, and included PubMed, Scopus, Embase, Google Scholar, Web of Science, CINAHL, and The Cochrane Library. The study designs that were included in this review are posters, abstracts, conference proceedings, case reports, case series, cohort, and randomized trials. The searches were conducted by combining selected MeSH terms, keywords, and phrases to achieve maximum sensitivity. An example of search strategy used in PubMed is as follows: ((“"sleep" OR ("Sleep Apnea, Obstructive"[Mesh])) OR ((“Therapeutics"[Mesh]) OR (“Complementary Therapies"[Mesh]))) AND (("Provent") OR ("nasal expiratory positive airway pressure" OR ("nasal EPAP”)))). Conference proceedings, nasal EPAP patent publications, and references of relevant articles were also searched to minimize the risk of missing potentially relevant publications. Also clinical trial websites were searched using appropriate terms; only one clinical trial was found which is recently completed but not published yet.

Data was abstracted in blinded manner, and reviewers (MR and MC) agreed on the included studies. Articles were selected using preset inclusion and exclusion criteria and studies were included for the review by the consensus of authors (MR and MC). Inclusion criteria, (1) adult OSA patients who underwent treatment with nasal EPAP or Provent therapy with quantitative outcome data such as apnea-hypopnea index (AHI), quality of life questionnaires, or Epworth Sleepiness Scale (ESS) before and after treatment, (2) studies with the objective of evaluating the effectiveness of nasal EPAP, and (3) all languages, were included. Exclusion criteria were as follows: (1) studies using Theravent as a snore prevention device, (2) patients under 18 years of age, or (3) no clear outcomes such as AHI and ESS reported. The metaanalysis was only performed for those studies, which reported means and standard deviations. The corresponding authors for studies reporting median values were contacted twice, and if the means and standard deviations could not be obtained, then the studies were included in the systematic review but were excluded from this meta-analysis.

The primary outcomes reviewed in the meta-analysis included the AHI, respiratory disturbance index (RDI), oxygen desaturation index (ODI), ESS, and quality of life questionnaires to assess the efficacy of nasal EPAP. Secondary outcomes included adherence or any other outcome reported by included studies. The National Institute for Health and Clinical Excellence (NICE) was used to assess the quality of studies. The Preferred Reporting Items for Systematic Reviews and Meta-Analyses (PRISMA) statement was utilized throughout this systematic review and meta-analysis [9].

2.1. Statistical Analysis. Statistical evaluation was performed using Review Manager (REVMAN) software version 5.3 (Copenhagen: The Nordic Cochrane Centre, The Cochrane Collaboration, 2014). The pre- and post-nasal EPAP means, standard deviations (SD), mean differences (MD), 95\% confidence intervals (CI), and $p$ values were calculated using the IBM Statistical Package for Social Sciences (SPSS) software, version 20.0 (Armonk, New York, USA). Combined mean differences and 95\% CI were calculated for studies reporting means and SD. Combined mean differences were not calculated for studies who did not provide standard deviations or median values; instead data was manually entered into the table. The null hypothesis for this study was that there is no difference between polysomnographic outcomes and ESS pre- and post-nasal EPAP therapy. Random effects modeling was used for analysis. Bias was analyzed by evaluating the funnel plots created by REVMAN. Inconsistency was evaluated using $I^{2}$ statistic (low: 25\%, moderate: 50\%, and high: $75 \%$ ) [10]. The Cochran $Q$ statistic $p$ value $\leq 0.10$ was considered statistically significant heterogeneity based on published guidelines [11]. For each variable evaluated (AHI, ESS, etc.) a sensitivity analysis was performed if there was inconsistency or heterogeneity in order to determine which study was the cause; this was performed by using REVMAN and removing one study at a time until no inconsistency or heterogeneity was present.

\section{Results}

The searches yielded a total of fifty-six articles after exclusion of duplicates, which were screened. Of these articles, thirty of them were potentially relevant and the full-text versions were downloaded for detailed evaluation (Figure 1). Eight of these were review articles discussing nasal EPAP alternative as treatment for OSA, and one was a case report discussing the emergence of complex sleep apnea with nasal EPAP use. After detailed review, the authors agreed that eighteen studies (eight original studies [12-19] and ten conference papers [2029]) met the inclusion and exclusion criteria. The combined studies had 920 patients, mean age $50.2 \pm 12.7$ years, with an average body mass index (BMI) of $32.2 \pm 6.7 \mathrm{~kg} / \mathrm{m}^{2}$. The earliest published study was by Colrain et al. in 2008 and Friedman et al. published the most recent study in 2015. 


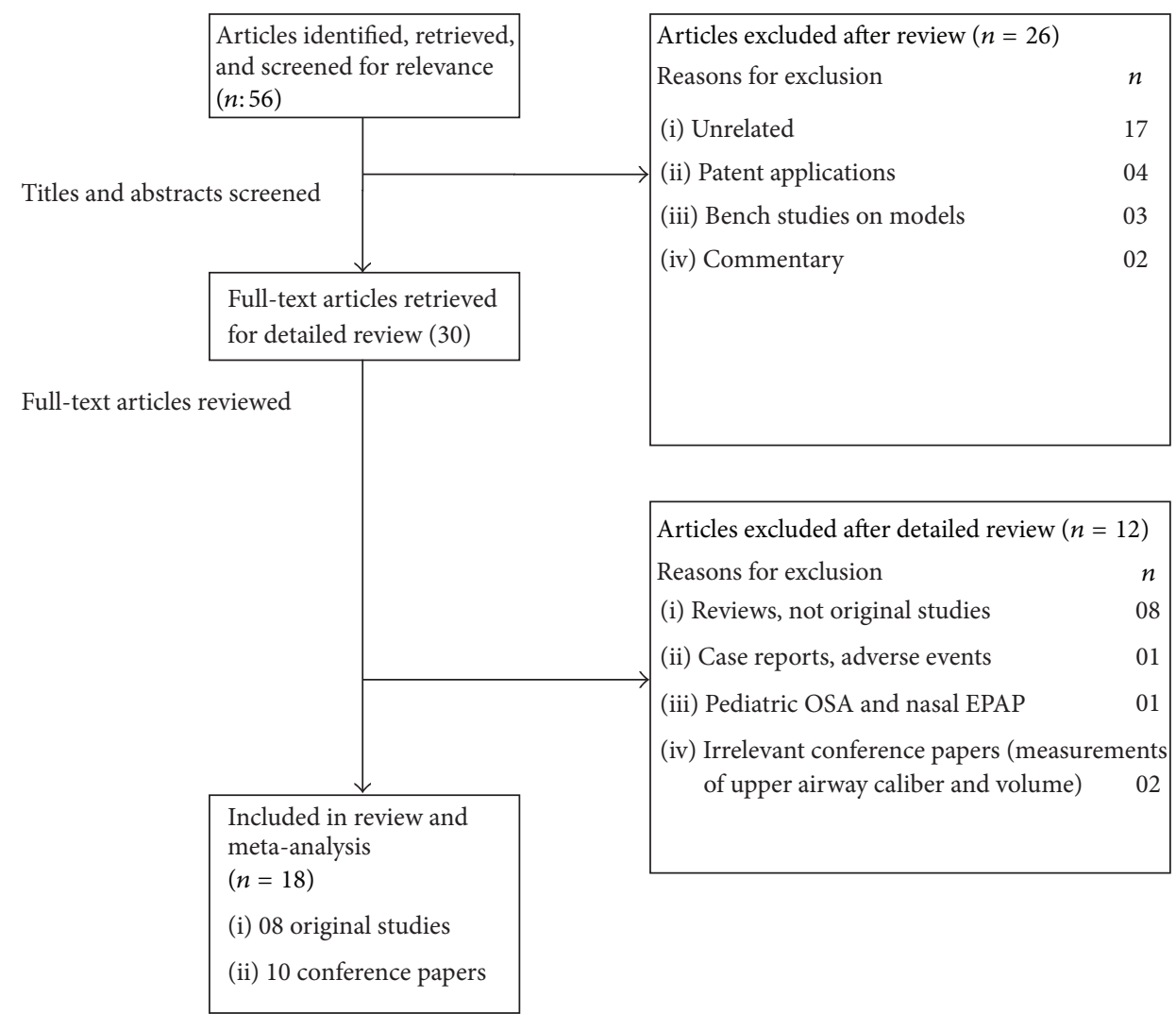

Figure 1: Nasal EPAP devices study selection flowchart.

The outcomes analyzed by these studies included AHI, ODI, minimum oxygen saturation $\left(\mathrm{SPO}_{2}\right)$, mean $\mathrm{SPO}_{2}$, percent of total sleep time (\%TST) with $\mathrm{SPO}_{2}<90 \%$, critical closing pressure $\left(P_{\text {crit }}\right)$, end tidal $\mathrm{CO}_{2}\left(\mathrm{EtCO}_{2}\right)$, lung volumes, blood pressure (BP), and quality of life questionnaires including Pittsburgh Sleep Quality Index (PSQI), Functional Outcomes of Sleep Questionnaire (FOSQ), and ESS.

All original studies underwent a quality assessment using the NICE quality assessment tool, and most of these studies were prospective with one dual-center and one multicenter randomized clinical trial (Table 1). The study quality assessment is presented in Table 2. Overall, the quality of original included studies was high and majority of them met 6-7 out of 8 criteria items evaluated by the quality assessment tool.

\section{General Characteristics of Included Studies}

The follow-up data was highly variable ranging from overnight study to a 12-month follow-up. Six original studies reported nasal EPAP to be effective in reducing AHI and other OSA related parameters. One randomized dual-center placebo-controlled trial failed to show efficacy of nasal EPAP and the majority of patients in this trial had elevated AHI and ESS (Table 3) and LSAT and ODI (Table 4). This study by Rossi et al. included OSA patients who were previously optimally treated on CPAP and were randomized into CPAP, Placebo-Provent, and Provent for 2 weeks and then were tested for OSA measures including AHI, ODI, and ESS as well as diastolic blood pressure. These patients were optimally treated with CPAP prior to randomization. This study reported higher residual AHI as well as diastolic blood pressure in Placebo and Provent group after CPAP withdrawal. Studies reporting adherence had an overall high nasal EPAP use, ranging between 80 and $94 \%$.

\section{Treatment Effect Data}

5.1. Apnea-Hypopnea Index. Polysomnography outcomes for nasal EPAP in 345 patients demonstrated that the AHI decreased from an overall mean $(\mathrm{M}) \pm$ standard deviation $(\mathrm{SD})$ in 345 patients from $27.32 \pm 22.24$ to $12.78 \pm 16.89$ events/hr (relative reduction $=53.2 \%$ ); see Table 3 . A subanalysis for these studies was performed and the AHI mean difference was -14.78 events/hr [95\% CI -19.12, -10.45], overall effect $Z=6.69, p$ value $<0.00001, Q$ statistic $p$ value $=0.0002$ (significant heterogeneity), and $I^{2}=72 \%$ (high inconsistency); see Figure 2. The funnel plot for AHI MD was scattered and only moderately distributed into an inverted funnel shape suggesting moderate publication bias. The sensitivity analysis demonstrated that the studies by Mansfield et al. and Walsh et al. contributed to the heterogeneity and inconsistency and, after their removal from the meta-analysis, there was no significant heterogeneity $(Q$ statistic $p$ value $=0.97)$ and no inconsistency $\left(I^{2}=0 \%\right)$. 


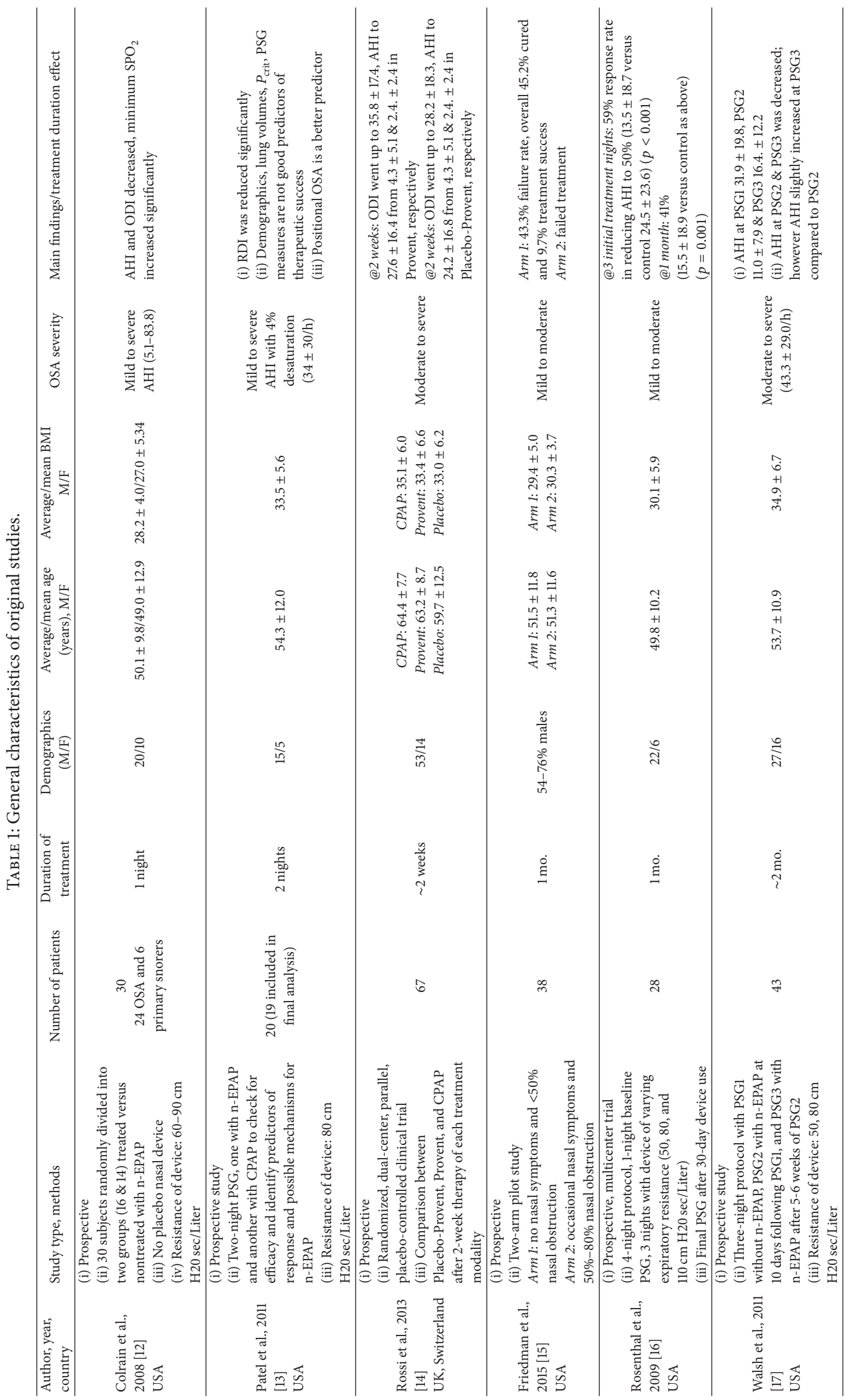




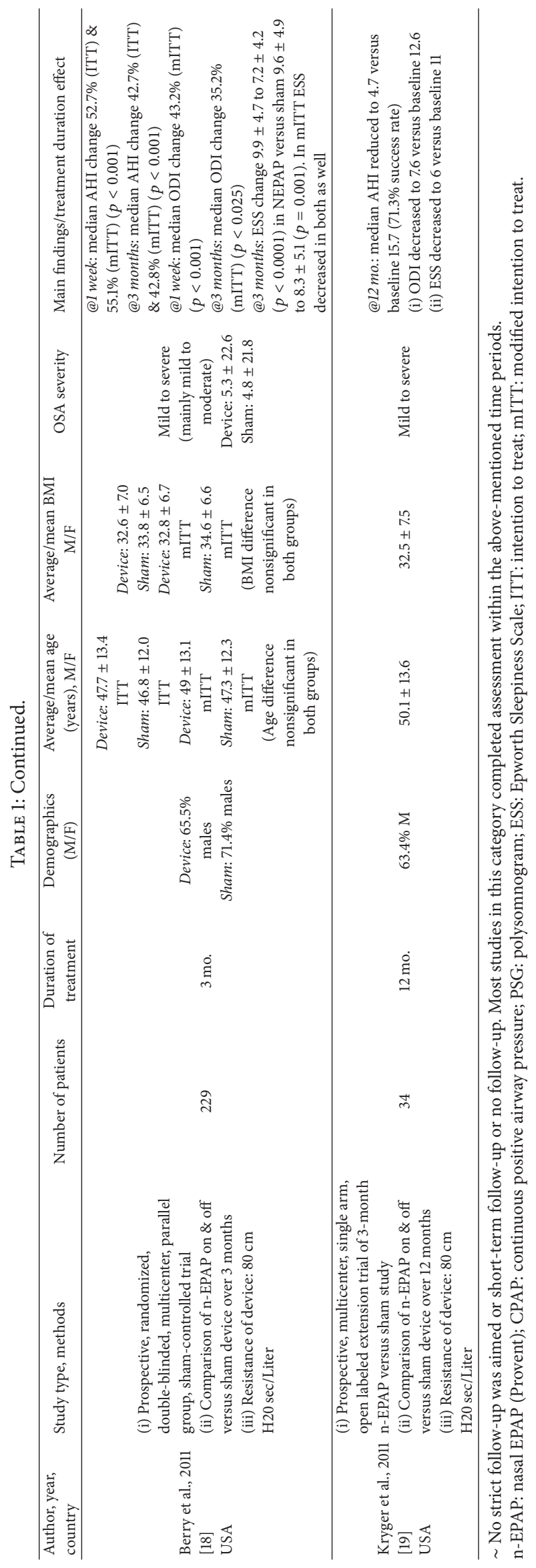




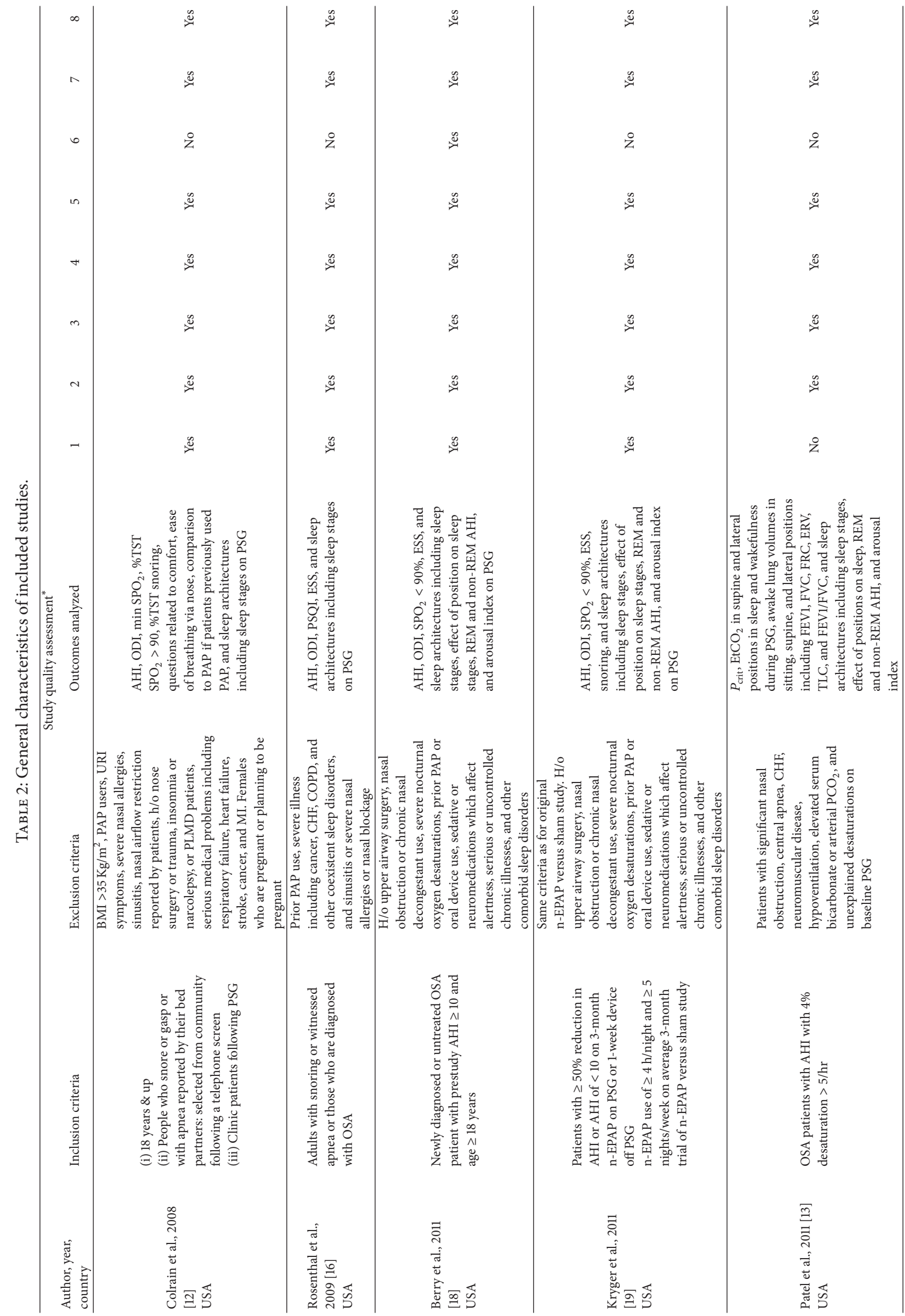




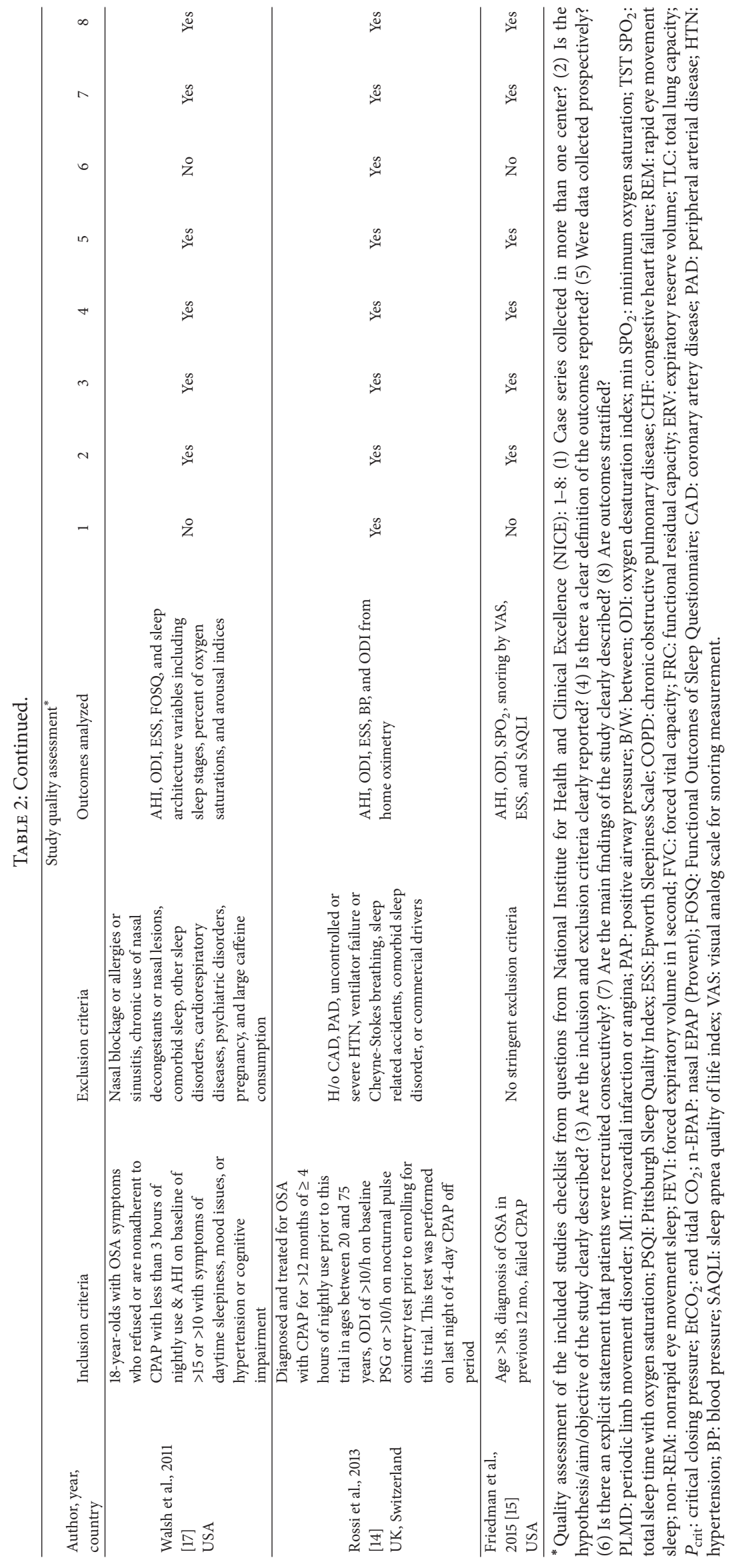




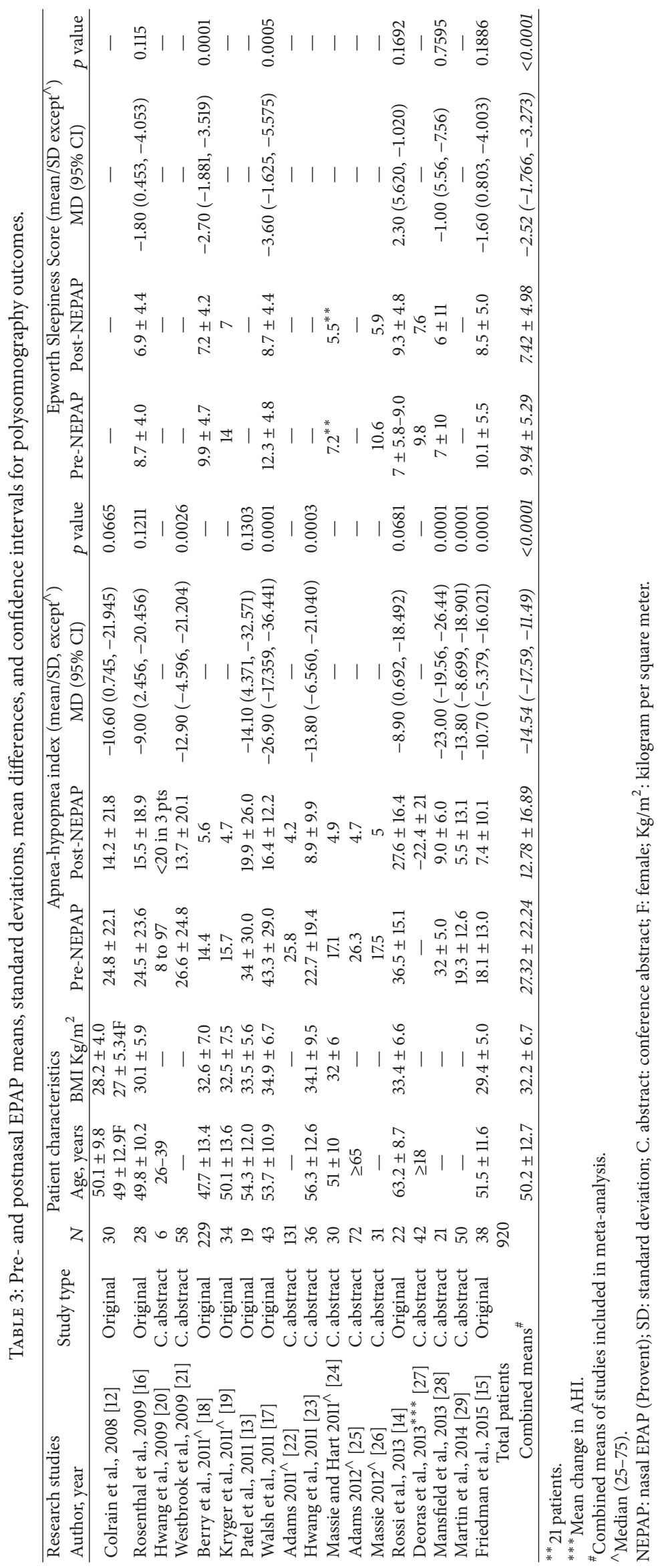




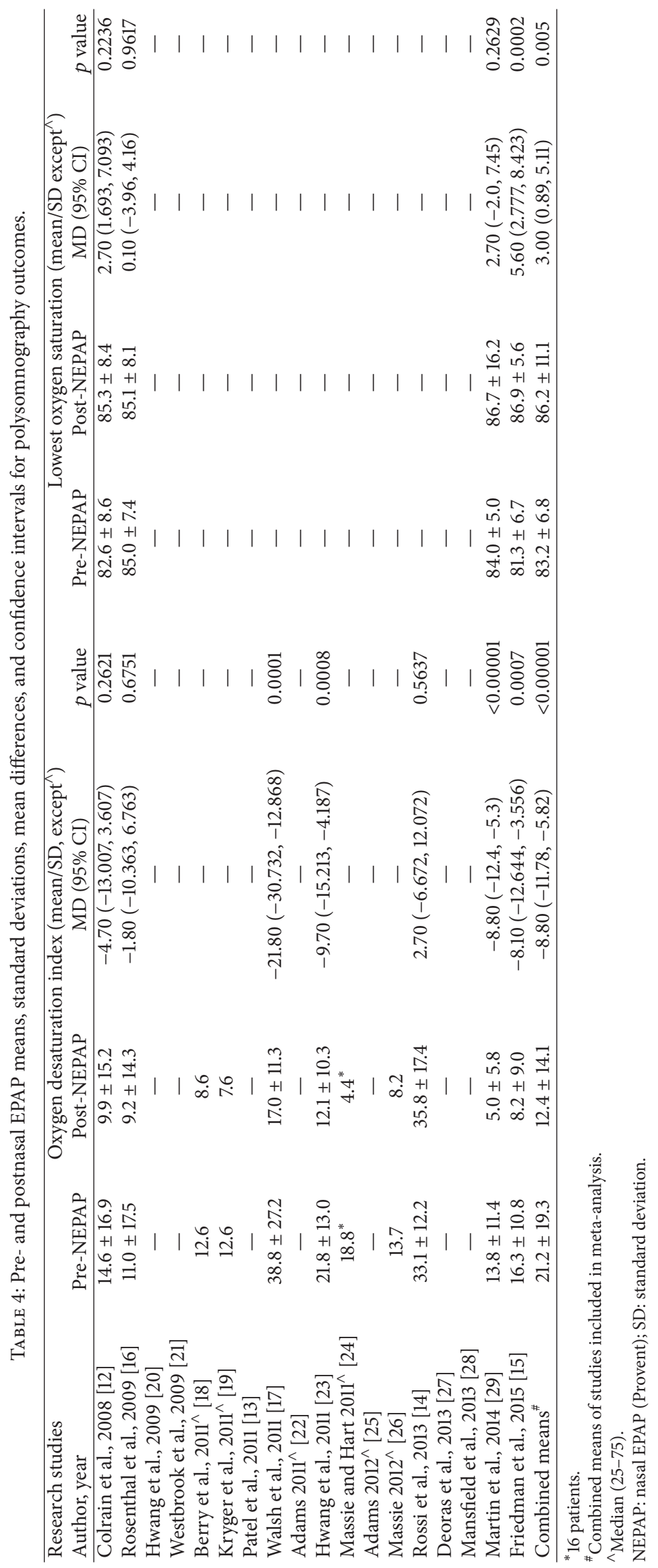




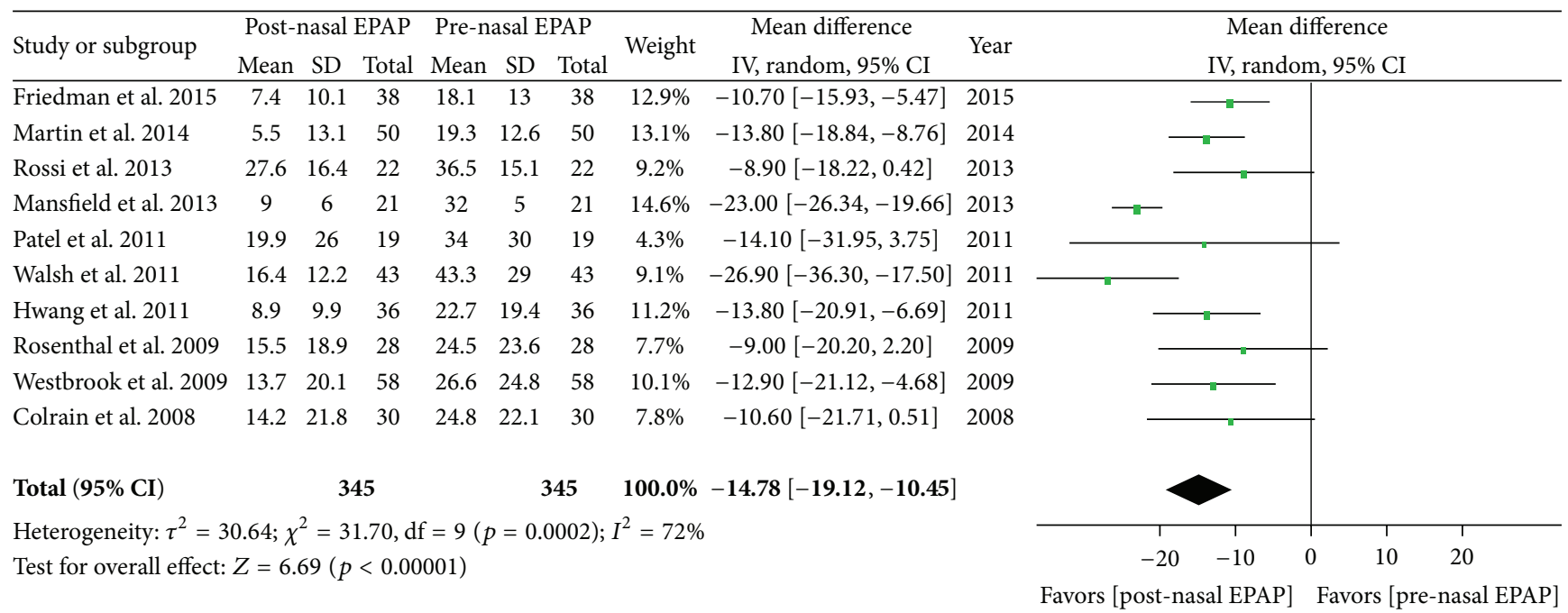

FIGURE 2: Pre- and post-nasal EPAP therapy outcomes for apnea-hypopnea index (events per hour), mean difference. SD: standard deviation; CI: confidence interval; nasal EPAP device: nasal expiratory positive airway pressure device (Provent).

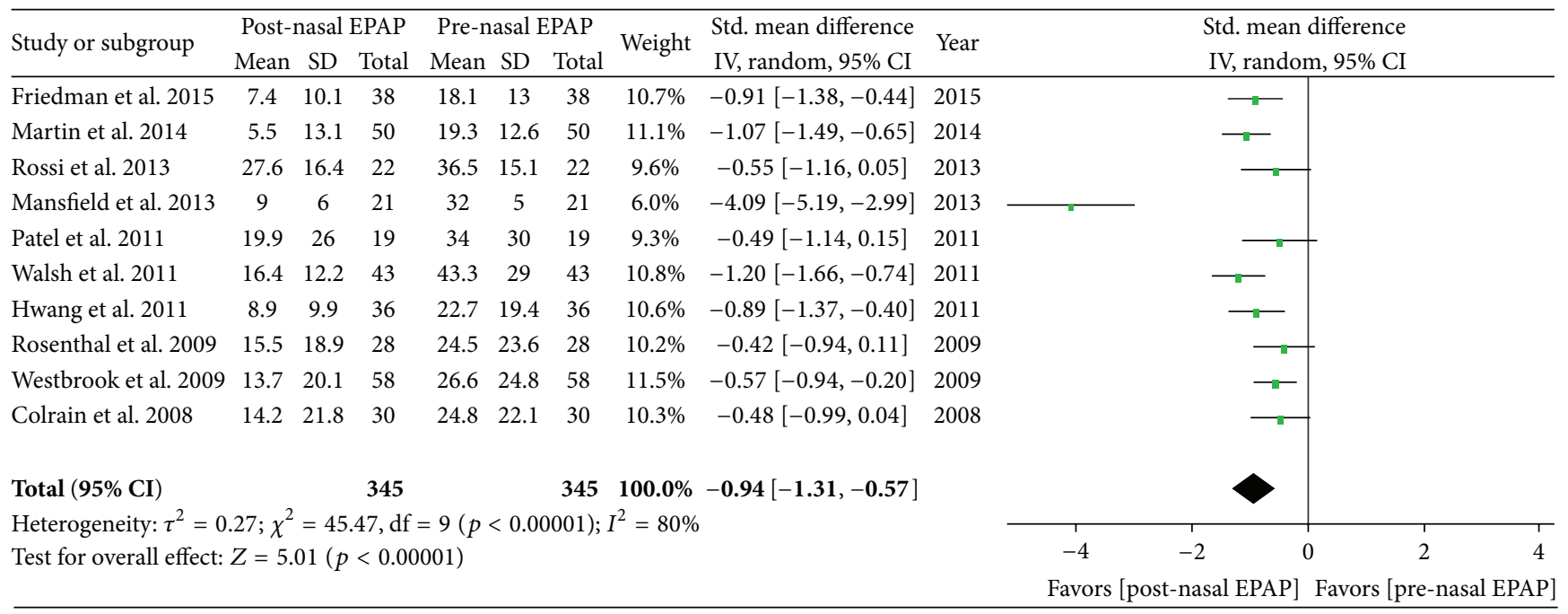

FIGURE 3: Pre- and post-nasal EPAP therapy outcomes for apnea-hypopnea index (events per hour), standardized mean difference. SD: standard deviation; CI: confidence interval; nasal EPAP device: nasal expiratory positive airway pressure device (Provent).

For all 345 patients, the AHI standardized mean difference (SMD) was -0.94 [95\% CI -1.31, -0.57] (large magnitude of effect), overall effect $Z=5.01, p$ value $<0.00001, Q$ statistic $p$ value $<0.00001$ (significant heterogeneity), and $I^{2}=80 \%$ (high inconsistency); see Figure 3.

5.2. Oxygen Desaturation Index. Polysomnography outcomes for nasal EPAP demonstrated that the ODI decreased from an overall $\mathrm{M} \pm \mathrm{SD}$ of $21.2 \pm 19.3$ to $12.4 \pm 14.1$ events $/ \mathrm{hr}$ (relative reduction $=41.5 \%$ ); see Table 4 . A subanalysis using random effects modeling was performed for seven studies (247 patients) in which $\mathrm{M} \pm \mathrm{SD}$ were reported, and the ODI mean difference was -7.69 events/hr $[95 \% \mathrm{CI}-11.78,-3.60]$, overall effect $Z=3.68, p$ value $=0.0002, Q$ statistic $p$ value $=$ 0.005 (significant heterogeneity), and $I^{2}=67 \%$ (moderate inconsistency); see Figure 4. The funnel plot for ODI MD was distributed evenly into an inverted funnel shape, suggesting no publication bias. The sensitivity analysis was performed and studies by Walsh et al. and Rossi et al. were found to be the sources of heterogeneity; after the removal of those two studies, there was no significant heterogeneity ( $Q$ statistic $p$ value $=0.51)$ and no inconsistency $\left(I^{2}=0 \%\right)$. For all 247 patients, the ODI SMD was -0.58 [95\% CI $-0.91,-0.25$ ] (medium magnitude of effect), overall effect $Z=3.42, p=$ $0.0006, Q$ statistic $p$ value $=0.004$ (significant heterogeneity), and $I^{2}=69 \%$ (moderate inconsistency).

5.3. Lowest Oxygen Saturation. Polysomnography outcomes for nasal EPAP demonstrated that the minimum $\mathrm{SPO}_{2}$ improved from an overall $\mathrm{M} \pm \mathrm{SD}$ of $83.2 \pm 6.8 \%$ to 


\begin{tabular}{|c|c|c|c|c|c|c|c|c|c|c|c|c|c|c|}
\hline \multirow{3}{*}{$\begin{array}{l}\text { Study or subgroup } \\
\text { Friedman et al. } 2015\end{array}$} & \multicolumn{3}{|c|}{ Post-nasal EPAP } & \multicolumn{3}{|c|}{ Pre-nasal EPAP } & \multirow{3}{*}{$\begin{array}{c}\text { Weight } \\
18.1 \%\end{array}$} & \multirow{3}{*}{$\begin{array}{c}\text { Mean difference } \\
\text { IV, random, 95\% CI } \\
-8.10[-12.57,-3.63]\end{array}$} & \multirow{3}{*}{$\begin{array}{c}\text { Year } \\
2015\end{array}$} & \multirow{2}{*}{\multicolumn{5}{|c|}{$\begin{array}{l}\text { Mean difference } \\
\text { IV, random, 95\% CI }\end{array}$}} \\
\hline & \multirow{2}{*}{$\begin{array}{c}\text { Mean } \\
8.2\end{array}$} & \multirow{2}{*}{$\frac{\mathrm{SD}}{9}$} & \multirow{2}{*}{$\begin{array}{c}\text { Total } \\
38\end{array}$} & \multirow{2}{*}{$\begin{array}{c}\text { Mean } \\
16.3\end{array}$} & \multirow{2}{*}{$\frac{S D}{10.8}$} & \multirow{2}{*}{$\frac{\text { Total }}{38}$} & & & & & & & & \\
\hline & & & & & & & & & & & & & & \\
\hline Martin et al. 2014 & 5 & 5.8 & 50 & 13.8 & 11.4 & 50 & $19.6 \%$ & $-8.80[-12.35,-5.25]$ & 2014 & & & & & \\
\hline Rossi et al. 2013 & 35.8 & 17.4 & 22 & 33.1 & 12.2 & 22 & $11.0 \%$ & $2.70[-6.18,11.58]$ & 2013 & & & & & \\
\hline Hwang et al. 2011 & 12.1 & 10.3 & 36 & 21.8 & 13 & 36 & $16.4 \%$ & $-9.70[-15.12,-4.28]$ & 2011 & & 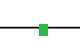 & & & \\
\hline Walsh et al. 2011 & 17 & 11.3 & 43 & 38.8 & 27.2 & 43 & $11.1 \%$ & $-21.80[-30.60,-13.00]$ & 2011 & & & & & \\
\hline Rosenthal et al. 2009 & 9.2 & 14.3 & 28 & 11 & 17.5 & 28 & $11.7 \%$ & $-1.80[-10.17,6.57]$ & 2009 & & & & & \\
\hline Colrain et al. 2008 & 9.9 & 15.2 & 30 & 14.6 & 16.9 & 30 & $12.0 \%$ & $-4.70[-12.83,3.43]$ & 2008 & & & & - & \\
\hline Total $(95 \% \mathrm{CI})$ & & & 247 & & & 247 & $100.0 \%$ & $-7.69[-11.78,-3.60]$ & & & & & & \\
\hline \multirow{2}{*}{\multicolumn{10}{|c|}{$\begin{array}{l}\text { Heterogeneity: } \tau^{2}=18.92 ; \chi^{2}=18.32, \mathrm{df}=6(p=0.005) ; I^{2}=67 \% \\
\text { Test for overall effect: } Z=3.68(p=0.0002)\end{array}$}} & -20 & -10 & 0 & 10 & 20 \\
\hline & & & & & & & & & & Favors $[\mathrm{p}$ & -nasal I & & Favors [pre & sal EPAP] \\
\hline
\end{tabular}

FIgURE 4: Pre- and post-nasal EPAP therapy outcomes for oxygen desaturation index (events per hour), mean difference. SD: standard deviation; CI: confidence interval; nasal EPAP device: nasal expiratory positive airway pressure device (Provent).

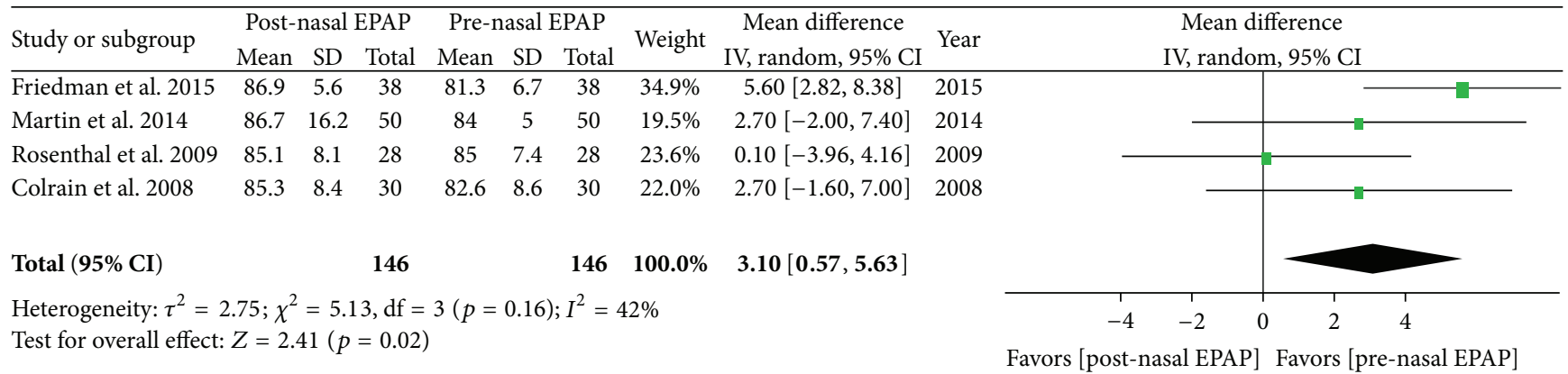

FIGURE 5: Pre- and post-nasal EPAP therapy outcomes for lowest oxygen saturation (percent), mean difference. SD: standard deviation; CI: confidence interval; nasal EPAP device: nasal expiratory positive airway pressure device (Provent).

$86.2 \pm 11.1 \%$ (3-point oxygenation increase); see Table 4. A subanalysis using random effects modeling was performed for four studies (146 patients) in which $\mathrm{M} \pm \mathrm{SD}$ were reported, and the minimum $\mathrm{SPO}_{2}$ mean difference was 3.10 oxygen saturation points [95\% CI $0.57,5.63]$, overall effect $Z=2.41$, $p$ value $=0.02, Q$ statistic $p$ value $=0.16$ (no statistically significant heterogeneity), and $I^{2}=42$ (low inconsistency) (Figure 5). Exclusion of the study by Friedman et al. resulted in no significant heterogeneity $(Q$ statistic $p$ value $=0.61)$ and no inconsistency $\left(I^{2}=0 \%\right)$. The funnel plot was not performed given that there were only four studies. The minimum $\mathrm{SPO}_{2}$ SMD was 0.37 [95\% CI 0.00, 0.73] (small magnitude of effect), overall effect $Z=1.97, p$ value $=0.05$, $Q$ statistic $p$ value $=0.07$ (significant heterogeneity), and $I^{2}=$ $58 \%$ (moderate inconsistency).

5.4. Epworth Sleepiness Scale. The sleepiness questionnaire for pre- and post-nasal EPAP demonstrated that the ESS improved from an overall $\mathrm{M} \pm \mathrm{SD}$ of $9.94 \pm 5.29$ to $7.42 \pm 4.98$, $p$ value $<0.0001$; see Table 3 . A subanalysis using random effects modeling was performed for five studies (359 patients) in which $\mathrm{M} \pm \mathrm{SD}$ were reported, and the ESS mean difference was -2.61 [95\% CI $-3.29,-1.94$ ], overall effect $Z=7.55$, $p$ value $<0.00001, Q$ statistic $p$ value $=0.64$ (no significant heterogeneity), and $I^{2}=0 \%$ (no inconsistency). The funnel plot for ESS MD was fairly evenly distributed into an inverted funnel shape, suggesting no publication bias. For all 359 patients, the ESS SMD was -0.52 [95\% CI -0.71, -0.33] (medium magnitude of effect), overall effect $Z=5.43, p$ value $<0.00001, Q$ statistic $p$ value $=0.30$ (no significant heterogeneity), and $I^{2}=18 \%$ (no inconsistency).

5.5. Snoring. Snoring was not assessed by all studies but reduction in snoring was uniformly seen in studies that assessed snoring (i.e., Colrain et al., Friedman et al., and Kryger et al.).

\section{Discussion}

Not all OSA treatments modalities are effective in controlling sleep apnea or improving quality of life as considerable variation exists among therapeutic modalities with respect to reductions in obstructive respiratory events $[30,31]$. This systematic review and meta-analysis investigated the effectiveness of nasal EPAP and found it to be a promising addition to the existing therapeutic treatment modalities for OSA. There are six main findings of this systematic review and meta-analysis worth noting. 
First, the majority of studies (including most conference abstracts) demonstrated an improvement in polysomnography respiratory parameters such as AHI, ODI, and LSAT. Overall, nasal EPAP (Provent) reduced AHI by 53.2\%, ODI by $41.5 \%$ and improved LSAT by 3 oxygen saturation points. Although AHI was reduced for most studies, the AHI reductions are generally less as compared to CPAP. It is well established that CPAP generally reduces AHI to less than 5 events/hr [4]. The results of nasal EPAP are however somewhat comparable for oral appliances or less invasive surgical procedures which are less effective than CPAP [32, 33]; nonetheless no head to head comparison has been done. Previous studies have compared oral appliances and surgeries with CPAP but studies for nasal EPAP have only compared it to CPAP. Perhaps future studies comparing nasal EPAP with both invasive/less invasive surgeries and oral appliances could be helpful to establish the success rate in each group given that optimal treatment of OSA is the key to prevent cardiovascular complications as even low AHI or mild OSA has been linked with cardiovascular outcomes [34].

Second, there were differences in study design and the length of follow-up which make it difficult to generalize the potential true long-term effectiveness of nasal EPAP. There was only one study (Kryger et al.) with 12-month followup which demonstrated maintenance of therapeutic effects in terms of AHI reduction, snoring, and subjective daytime sleepiness. Nevertheless, majority of studies have variable and inconsistent response to treatment with nasal EPAP and longer duration was inversely proportional to residual AHI for unclear reasons. This inconsistent response could be due to the fact that nasal EPAP increases lung volumes by creating positive airway pressure rather than exerting direct pressure at the upper airway like PAP devices. As OSA can be due to the collapse at any airway level, nasal EPAP may not be able to generate enough airway pressure to overcome airway collapse at all levels. In the aforementioned studies, different nasal EPAP devices with varying degrees of expiratory resistance were used without any significant differences in outcomes. The elimination of SDB is dependent on sustained end-expiratory pressures generated by these valves during different stages of sleep; therefore, it is likely that this end-expiratory pressure will be different from individual to individual with respect to different positions, stages of sleep, mouth breathing, nasal obstruction, and so forth. This was objectively demonstrated by Patel et al., where end-expiratory positive pressure varied widely among different nasal EPAP responders $(5-23 \mathrm{~cm}$ of water). Therefore, it is possible to titrate such patients in sleep lab to determine the lowest effective pressure in different stages/positions of sleep, which can help select an appropriate resistance for nasal EPAP device. Further studies are needed to explore this hypothesis.

Third, there was no clear predictor (demographic factors, medical history, or physical exam findings) as to which patients will respond most favorably to this device. Patel et al. specifically examined demographic factors that could predict therapeutic response to nasal EPAP. In their study, no significant association was found between the degrees of OSA severity and general characteristics of patients including age, BMI, gender, sleep stage dependent SDB, therapeutic
CPAP levels, lung volumes, and $P_{\text {crit }}$ values. Friedman et al. demonstrated that nasal EPAP may be useful in patients without significant nasal obstruction and daily nasal symptoms. Nevertheless, it is quite possible that patients with positional sleep apnea or those with only mild to moderate OSA or patients without significant nasal obstructions at baseline might benefit the most from this therapy. Treatment was not as successful in patients with severe OSA (Colrain et al.) or in those who had low baseline LSAT along with higher AHI (Patel et al., Berry et al., and Rosenthal et al.). An important confounder is that most studies had stringent inclusion and exclusion criteria and patients with severe comorbidities were also excluded which makes it more difficult to determine which patient profile best suites this therapy. Therefore, additional studies are needed not only to confirm which patient characteristics lead to a more favorable response, but also to stratify treatment effect on OSA related chronic comorbid diseases.

Fourth, Rossi et al. demonstrated a higher residual AHI as well as diastolic blood pressure in Placebo and Provent group after CPAP withdrawal. The high residual AHI in both arms of this study (Provent and Placebo-Provent) could be related to recruitment of subjects with higher baseline OSA severity (mean AHI of 38 events/h), when compared to Berry et al.s study (median AHI 13.8 events/hr in nasal EPAP versus 11.1 events/hr in sham) that showed preferable improvement with Provent therapy alone. Although the two arms in Rossi et al. study were very similar, the residual AHI and ODI after CPAP treatment as well as the four-night withdrawal rebound ODI values were slightly favorable in the PlaceboProvent arm compared to Provent arm. Also, it was not stated if patients had statistically significant difference in proportion to patients with positional OSA between the Placebo-Provent and Provent arm since this can independently influence residual AHI based on sleep position adopted during the night of sleep study.

Fifth, generally adherence was high for most studies that collected data for adherence (e.g., $84.2 \%$ in the study by Friedman, $80 \%$ by Wash, $88.2 \%$ by Berry, $89.3 \%$ by Kryger, $94 \%$ by Rosenthal, and $99 \%$ by Rossi et al.). Most studies did not report any serious adverse effects; however minor adverse events were common as much as in $42 \%$ of patients based on the longest duration study by Kryger et al. On searching literature, only one case report of treatment emergent central sleep apnea with nasal EPAP device was identified [35]. Common side effects observed in most studies include difficulty breathing, exhaling, and sleeping, dry mouth, nasal congestion/drainage/discomfort/itching, insomnia, and headache. Despite these adverse effects, adherence rates were not altered. It is however possible that this adherence might be artificially high as data was provided by subjective reports as compared to PAP where data downloads reflect objective adherence. Additionally, there was only one pediatric study which evaluated the efficacy of nasal EPAP in children 816 years old [36]. This was a small (14 subjects) clinical trial which reported significant improvement in AHI (nasal EPAP 0.6 versus placebo 4.2 ) but 3 subjects did not improve and 2 had worsening of OSA. Given that nasal EPAP has very limited data for children, it should be used cautiously, 
as other treatment options such as adenotonsillectomy, palate expansion, allergy management, and PAP therapy remain the gold standard.

Sixth, additional research is needed. Because it is not clear why some patients responded better to nasal EPAP than others, the stratification of patients based on additional findings or testing would be helpful. Recently, drug induced sleep endoscopy (DISE) studies have demonstrated that the anatomic location and the physiological pattern of collapse influence the baseline severity of OSA and the response to a particular treatment modality. About two-thirds of patients with OSA have a multilevel collapse, with the most common combination being palatal and tongue based collapse [37]. Nasal EPAP generates end-expiratory pressure leading to increase in functional residual capacity and tracheal traction and a trend towards increase in upper airway cross-sectional area [5]. On the other hand, expansion of the velopharyngeal airway volume during sleep is associated with improvement in OSA with use of oral appliances or oral pressure therapy $[38,39]$. Could the variable efficacy of nasal EPAP be related to its limited ability to overcome palatal and tongue base collapse, while having a favorable response in individuals with predominance of hypopharyngeal collapse? In the future, DISE and upper airway MRIs during sleep in subjects with OSA while using nasal EPAP may provide substantial information to explain such heterogeneity in treatment response. Another important variable that needs additional research is the effect of nasal cavity (i.e., inferior turbinate size [40], nasal septal deviations, and nasal septal perforations) and nasopharyngeal exam (adenoid hypertrophy) findings.

\section{Limitations}

The authors in this systematic review tried their best to identify all published as well as grey literature related to nasal EPAP; however it is possible that we failed to identify all relevant studies. It is also quite likely that studies that did demonstrate beneficial effect of nasal EPAP were never made for publication. Not all studies reported the same variables or means and hence were not included in the meta-analysis. The largest study by Berry et al. was not used for pooling random effects given that it did not report mean or standard deviations and instead the median data was provided (the corresponding author responded to our emails but raw data was not available). Similarly, many other studies reported data in median values, hence excluded from the meta-analysis portion of this review (Tables 3 and 4). Long-term followup data is limited for most studies with only two studies having 3-month and 12-month data. Data regarding preand posttreatment with nasal EPAP and BMI changes were not available for most studies. It is important to note that higher BMI is associated with complete concentric palatal collapse [37] and nasal EPAP may not be able to overcome the palatal collapse in all patients with same OSA severity but with different BMI. Given that pre- and post-BMI data is not available for most studies, it is possible that weight loss during the study period could have influenced the final outcome at the end of study. Many of the studies were industry funded; hence results are to be interpreted with caution.

\section{Conclusion}

Although nasal EPAP does not completely eliminate OSA, there is an improvement in OSA outcomes based on polysomnography and questionnaires (quality of life and sleepiness). The benefits include that the devices are highly portable and easy to use and there are no reported major side effects or complications reported from the use. For that reason, nasal EPAP might be an alternative for certain OSA patients who are either intolerant to PAP or in unusual circumstances without electricity or short trips away from home, and for those with mild or position dependent OSA without concurrent chronic medical problems. Further studies are needed to evaluate long-term efficacy and delineate clinical and polysomnographic profiles of patients who would be best suited for this therapy.

\section{Disclosure}

The authors do not have anything to disclose except that Dr. Clete A. Kushida received research and grant support from ResMed, Jawbone, Cephalon, Aerial BioPharma, and Impax Laboratories, worked as Consultant for Zephyr Sleep Technologies, Philips-Respironics, Morphy Smart Bed, and Nokia, and holds patent: Philips-Respironics. The study was not funded by industry.

\section{Disclaimer}

The views herein are the private views of the authors and do not reflect the official views of the Department of the Army or the Department of Defense.

\section{Conflict of Interests}

There is no conflict of interests regarding this paper.

\section{Acknowledgment}

The institution where the work was primarily performed is Sleep Disorders Center, University of Michigan, Ann Arbor 48109, USA.

\section{References}

[1] P. E. Peppard, T. Young, J. H. Barnet, M. Palta, E. W. Hagen, and K. M. Hla, "Increased prevalence of sleep-disordered breathing in adults," American Journal of Epidemiology, vol. 177, no. 9, pp. 1006-1014, 2013.

[2] C. E. Sullivan, F. Issa, M. Berthon-Jones, and L. Eves, "Reversal of obstructive sleep apnoea by continuous positive airway pressure applied through the nares," The Lancet, vol. 317, no. 8225, pp. 862-865, 1981.

[3] P. Gay, T. Weaver, D. Loube et al., "Evaluation of positive airway pressure treatment for sleep related breathing disorders in adults," Sleep, vol. 29, no. 3, pp. 381-401, 2006.

[4] T. E. Weaver and R. R. Grunstein, "Adherence to continuous positive airway pressure therapy: the challenge to effective treatment," Proceedings of the American Thoracic Society, vol. 5, no. 2, pp. 173-178, 2008. 
[5] C. W. Braga, Q. Chen, O. E. Burschtin, D. M. Rapoport, and I. Ayappa, "Changes in lung volume and upper airway using MRI during application of nasal expiratory positive airway pressure in patients with sleep-disordered breathing," Journal of Applied Physiology, vol. 111, no. 5, pp. 1400-1409, 2011.

[6] A. K. Mahadevia, E. Onal, and M. Lopata, "Effects of expiratory positive airway pressure on sleep-induced respiratory abnormalities in patients with hypersomnia-sleep apnea syndrome," American Review of Respiratory Disease, vol. 128, no. 4, pp. 708711, 1983.

[7] R. Heinzer, D. P. White, A. Malhotra et al., "Effect of expiratory positive airway pressure on sleep disordered breathing," Sleep, vol. 31, no. 3, pp. 429-432, 2008.

[8] M. Riaz, V. Certal, and M. Camacho, "Portable power supply options for positive airway pressure devices," Rural and Remote Health, vol. 15, no. 3, p. 3499, 2015.

[9] D. Moher, A. Liberati, J. Tetzlaff, and D. G. Altman, "Preferred reporting items for systematic reviews and meta-analyses: the PRISMA statement," International Journal of Surgery, vol. 8, no. 5, pp. 336-341, 2010.

[10] J. P. T. Higgins, S. G. Thompson, J. J. Deeks, and D. G. Altman, "Measuring inconsistency in meta-analyses," British Medical Journal, vol. 327, no. 7414, pp. 557-560, 2003.

[11] J. Lau, J. P. A. Ioannidis, and C. H. Schmid, "Quantitative synthesis in systematic reviews," Annals of Internal Medicine, vol. 127, no. 9, pp. 820-826, 1997.

[12] I. M. Colrain, S. Brooks, and J. Black, "A pilot evaluation of a nasal expiratory resistance device for the treatment of obstructive sleep apnea," Journal of Clinical Sleep Medicine, vol. 4, no. 5, pp. 426-433, 2008.

[13] A. V. Patel, D. Hwang, M. J. Masdeu, G.-M. Chen, D. M. Rapoport, and I. Ayappa, "Predictors of response to a nasal expiratory resistor device and its potential mechanisms of action for treatment of obstructive sleep apnea," Journal of Clinical Sleep Medicine, vol. 7, no. 1, pp. 13-22, 2011.

[14] V. A. Rossi, B. Winter, N. M. Rahman et al., "The effects of Provent on moderate to severe obstructive sleep apnoea during continuous positive airway pressure therapy withdrawal: a randomised controlled trial," Thorax, vol. 68, no. 9, pp. 854-859, 2013.

[15] M. Friedman, M. S. Hwang, S. Yalamanchali, T. Pott, M. Sidhu, and N. J. Joseph, "Provent therapy for obstructive sleep apnea: impact of nasal obstruction," The Laryngoscope, 2015.

[16] L. Rosenthal, C. A. Massie, D. C. Dolan, B. Loomas, J. Kram, and R. W. Hart, "A multicenter, prospective study of a novel nasal EPAP device in the treatment of obstructive sleep apnea: efficacy and 30-day adherence," Journal of Clinical Sleep Medicine, vol. 5, no. 6, pp. 532-537, 2009.

[17] J. K. Walsh, K. S. Griffin, E. H. Forst et al., "A convenient expiratory positive airway pressure nasal device for the treatment of sleep apnea in patients non-adherent with continuous positive airway pressure," Sleep Medicine, vol. 12, no. 2, pp. 147-152, 2011.

[18] R. B. Berry, M. H. Kryger, and C. A. Massie, "A novel nasal expiratory positive airway pressure (EPAP) device for the treatment of obstructive sleep apnea: a randomized controlled trial," Sleep, vol. 34, no. 4, pp. 479-485, 2011.

[19] M. H. Kryger, R. B. Berry, and C. A. Massie, "Long-term use of a nasal expiratory positive airway pressure (EPAP) device as a treatment for obstructive sleep apnea (OSA)," Journal of Clinical Sleep Medicine, vol. 7, no. 5, pp. 449-453, 2011.
[20] D. Hwang, A. Patel, G. Chen, I. Ayappa, and D. Rapoport, "Nasal epap-physiologic mechanism of action," in Proceedings of the 23rd Annual Meeting of the Associated Professional Sleep Societies (SLEEP '09), Abstract 0591, Seattle, Wash, USA, June 2009.

[21] P. Westbrook, R. Doshi, and B. Loomas, "Success rates of nasal expiratory positive airway pressure (NEPAP) via expiratory resistive load for the treatment of obstructive sleep apnea," in Proceedings of the 23rd Annual Meeting of the Associated Professional Sleep Societies (SLEEP '09), Seattle, Wash, USA, June 2009.

[22] G. Adams, "Retrospective case series analysis of a nasal expiratory positive airway pressure (EPAP) device to treat obstructive sleep apnea in a clinical practice," in Proceedings of the 25th Anniversary Meeting of the Associated Professional Sleep Societies (SLEEP '11), Minneapolis, Minn, USA, June 2011.

[23] D. Hwang, J. Chang, L. Gonzalez, E. Wigler, and K. Becker, "Nasal EPAP as a major osa therapeutic option in a clinical sleep center setting," in Proceedings of the 25th Annual Meeting Associated Professional Sleep Societies (SLEEP '11), Minneapolis, Minn, USA, June 2011.

[24] C. Massie and R. Hart, "Clinical efficacy of a nasal expiratory positive airway pressure (EPAP) device for the treatment of obstructive sleep apnea (OSA)," in Proceedings of the 25th Annual Meeting of the Associated Professional Sleep Societies (SLEEP '11), Minneapolis, Minn, USA, June 2011.

[25] G. Adams, "Nasal expiratory positive airway pressure (EPAP) device to treat obstructive sleep apnea in patients age 65 and over," in Proceedings of the 26th Annual Meeting of the Associated Professional Sleep Societies (SLEEP '12), Boston, Mass, USA, June 2012.

[26] C. Massie, "An analysis of responders to nasal expiratory positive airway pressure(EPAP) therapy during long term follow up," in Proceedings of the 26th Annual Meeting of the Associated Professional Sleep Societies (SLEEP '12), Boston, Mass, USA, June 2012.

[27] M. Deoras, J. Golish, D. Thornton et al., "Is nasal EPAP combined with nonsupine sleeping position effective in controlling OSA in those who have failed traditional treatment?" in Proceedings of the 27th Annual Meeting of the Associated Professional Sleep Societies (SLEEP '13), Baltimore, Md, USA, June 2013.

[28] D. Mansfield, G. Hamilton, and F. Tabart, "An expiratory positive airway pressure valve applied nasally (provent TM) improves polysomnographic indices of obstructive sleep apnea severity in a clinical practice case series," in Proceedings of the Sleep and Biological Rhythms, p. 50, Brisbane, Australia, 2013.

[29] I. D. Martin, M. D. Higgins, and S. Prasad, "Treatment of obstructive sleep apnea with nasal expiratory positive airway pressure (EPAP) devices: a retrospective analysis of 50 consecutive patients treated with combined conservative therapy," in Proceedings of the 28th Annual Meeting of the Associated Professional Sleep Societies (SLEEP '14), Minneapolis, Minn, USA, June 2014.

[30] V. Hoffstein, "Review of oral appliances for treatment of sleepdisordered breathing," Sleep and Breathing, vol. 11, no. 1, pp. 122, 2007.

[31] S. R. Patel, D. P. White, A. Malhotra, M. L. Stanchina, and N. T. Ayas, "Continuous positive airway pressure therapy for treating sleepiness in a diverse population with obstructive sleep apnea results of a meta-analysis," Archives of Internal Medicine, vol. 163, no. 5, pp. 565-571, 2003. 
[32] M. Barnes, R. D. McEvoy, S. Banks et al., "Efficacy of positive airway pressure and oral appliance in mild to moderate obstructive sleep apnea," American Journal of Respiratory and Critical Care Medicine, vol. 170, no. 6, pp. 656-664, 2004.

[33] A. G. Elshaug, J. R. Moss, A. M. Southcott, and J. E. Hiller, "Redefining success in airway surgery for obstructive sleep apnea: a meta analysis and synthesis of the evidence," Sleep, vol. 30, no. 4, pp. 461-467, 2007.

[34] Y. Peker, J. Hedner, J. Norum, H. Kraiczi, and J. Carlson, "Increased incidence of cardiovascular disease in middle-aged men with obstructive sleep apnea: a 7-year follow-up," American Journal of Respiratory and Critical Care Medicine, vol. 166, no. 2, pp. 159-165, 2002.

[35] A. Chopra, P. Das, K. Ramar, B. Staats, and E. K. St Louis, "Complex sleep apnea associated with use of nasal expiratory positive airway (nEPAP) device," Journal of Clinical Sleep Medicine, vol. 10, no. 5, pp. 577-579, 2014.

[36] S. A. Kureshi, P. R. Gallagher, J. M. McDonough et al., "Pilot study of nasal expiratory positive airway pressure devices for the treatment of childhood obstructive sleep apnea syndrome," Journal of Clinical Sleep Medicine, vol. 10, no. 6, pp. 663-669, 2014.

[37] A. V. Vroegop, O. M. Vanderveken, A. N. Boudewyns et al., "Drug-induced sleep endoscopy in sleep-disordered breathing: report on 1,249 cases," Laryngoscope, vol. 124, no. 3, pp. 797-802, 2014.

[38] A. S. L. Chan, K. Sutherland, R. J. Schwab et al., "The effect of mandibular advancement on upper airway structure in obstructive sleep apnoea," Thorax, vol. 65, no. 8, pp. 726-732, 2010.

[39] R. J. Schwab, C. Kim, L. Siegel et al., "Examining the mechanism of action of a new device using oral pressure therapy for the treatment of obstructive sleep apnea," Sleep, vol. 37, no. 7, pp. 1237-1247, 2014.

[40] M. Camacho, S. Zaghi, V. Certal et al., "Inferior turbinate classification system, grades 1 to 4: development and validation study," The Laryngoscope, vol. 125, no. 2, pp. 296-302, 2015. 


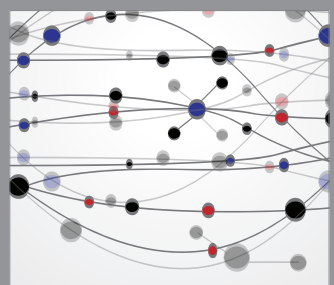

The Scientific World Journal
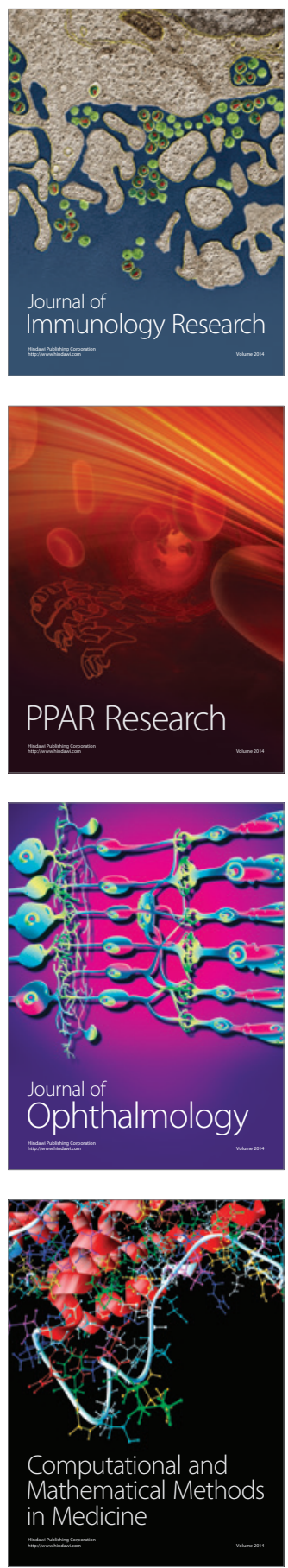

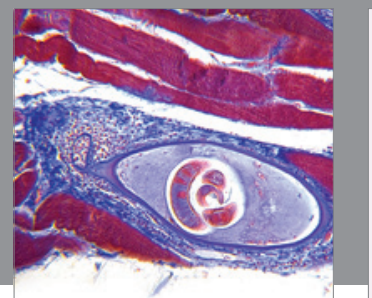

Gastroenterology

Research and Practice
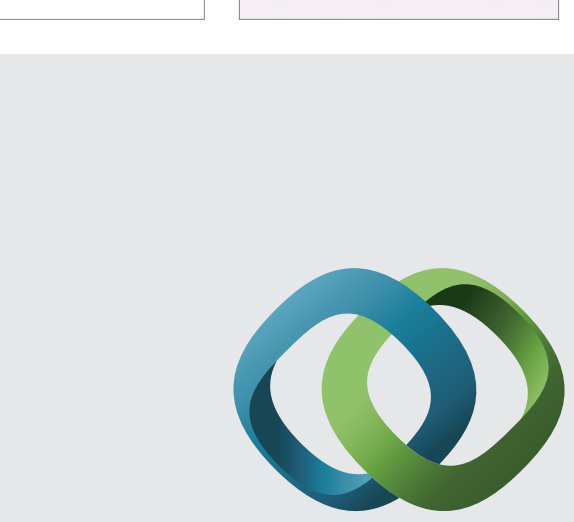

\section{Hindawi}

Submit your manuscripts at

http://www.hindawi.com
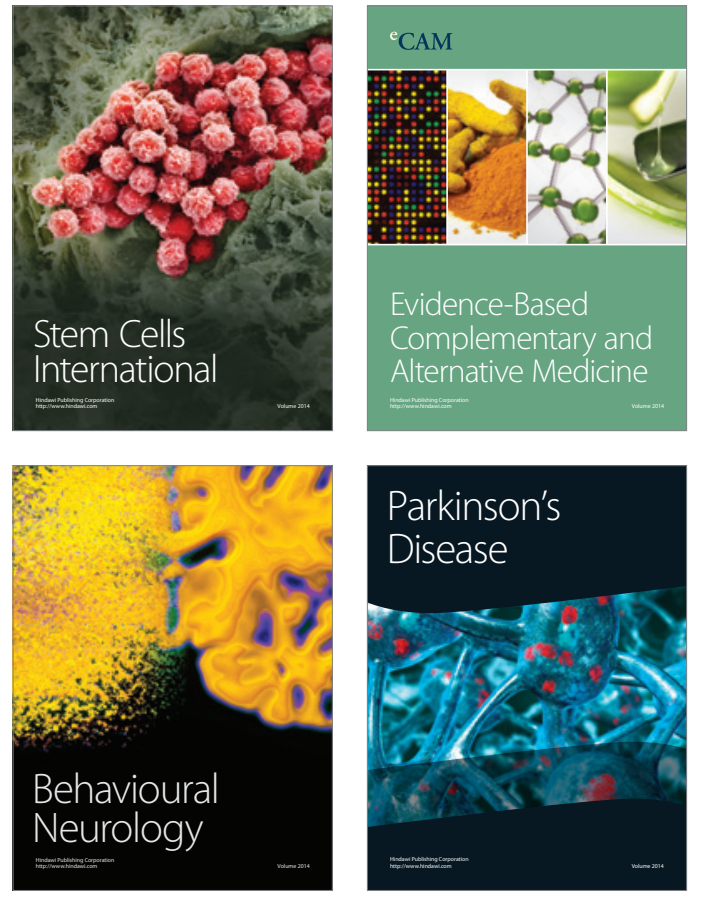
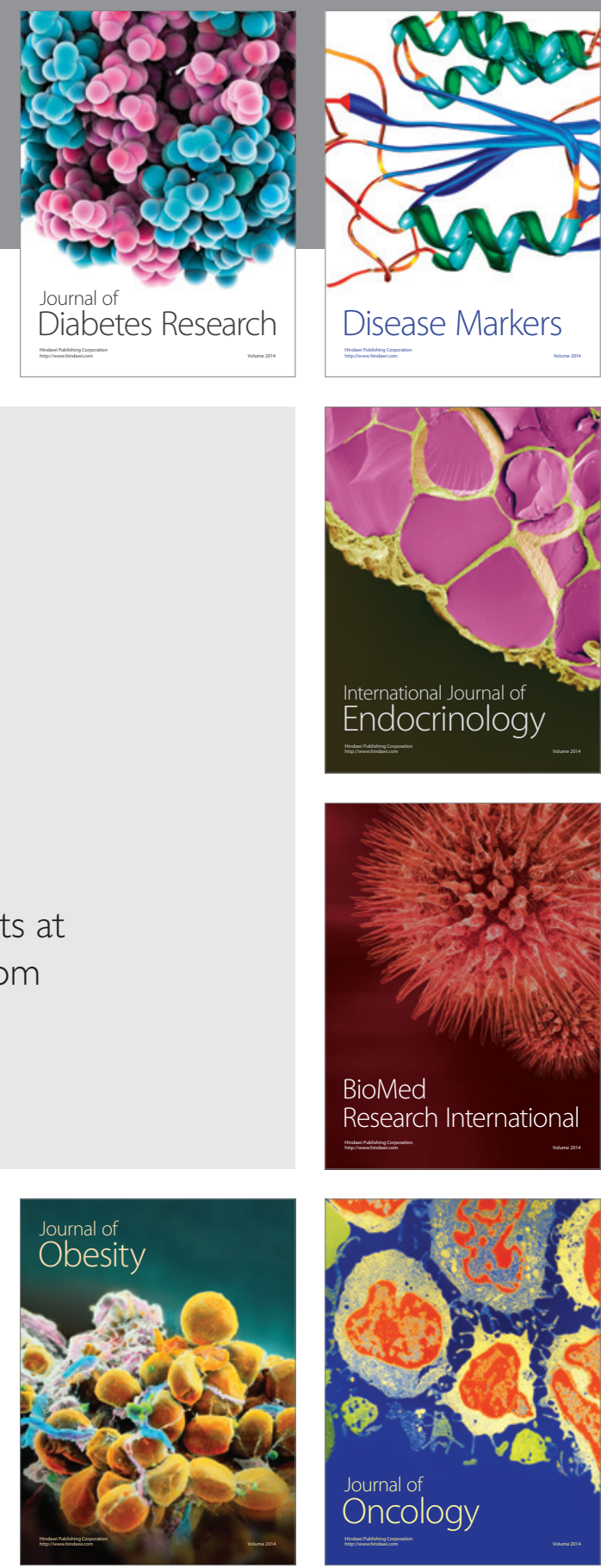

Disease Markers
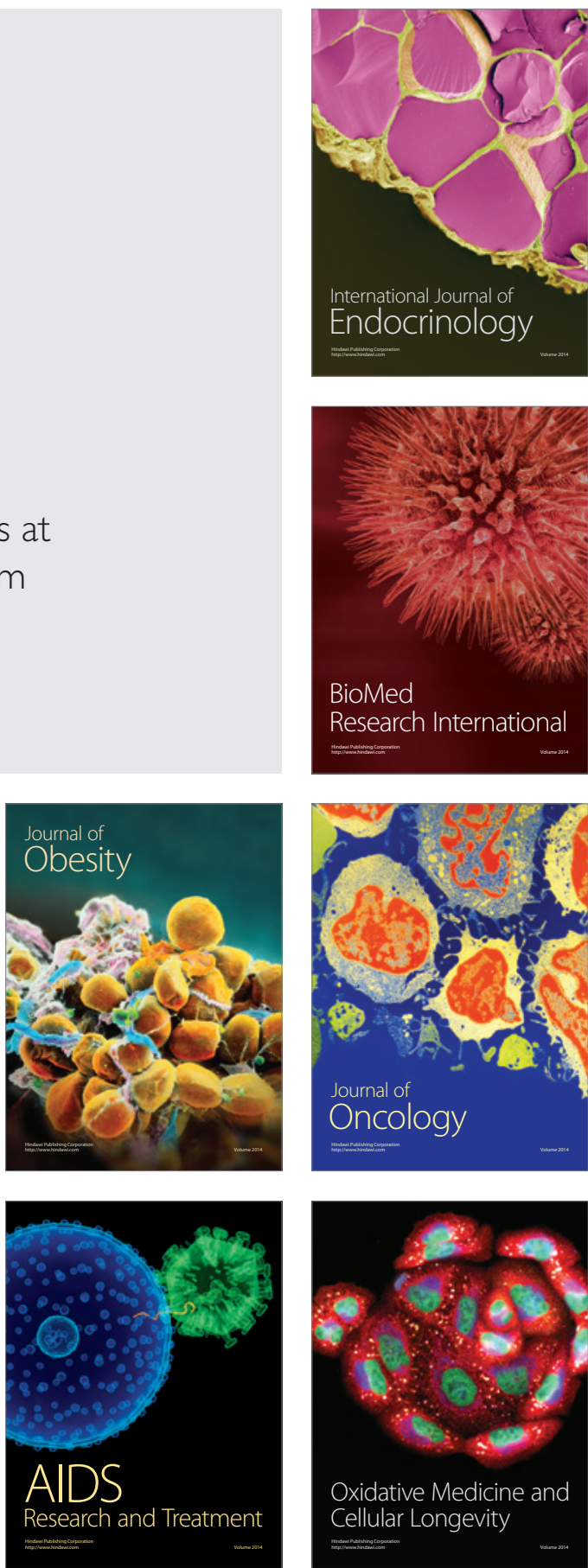